\title{
Les patients
}

\section{experts \\ dans la lutte \\ contre le Sida \\ au Cambodge}

Anne-Marie Moulin

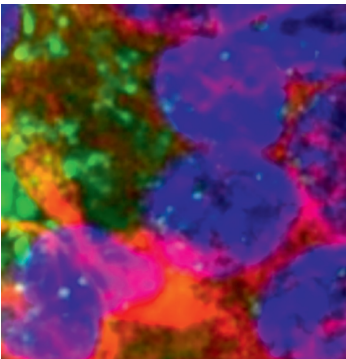

CNRS UMR SPHERE 7219

Université Paris 7, bâtiment Condorcet 4, rue Elsa Morante, 75013 Paris, France.

anne.saintromain@gmail.com

sont devenus un intermédiaire incontournable auprès de populations désemparées et peu informées et un rouage essentiel du fonctionnement des ONG (organisations non gouvernementales). Volant en aide à des gouvernements souvent peu actifs, dépourvus de moyens et/ou corrompus, les associations ont été de plus en plus considérées comme le premier échelon d'une démocratie idéale devenue l'étalon politique universel.

L'ouvrage d'Ève Bureau-Point [2] est consacré à la lutte contre le Sida au Cambodge, souvent présentée comme une Success Story. Success Story évidemment toute relative! Depuis le dépistage du premier cas de VIH en 1991, après la fin des Khmers rouges et de l'occupation vietnamienne, l'épidémie a flambé (la plus haute prévalence d'Asie en 1997, 3,5\% !), au moment où la paix faisait ses premiers pas et où l'aide internationale affluait dans le pays. C'est dans ce contexte que les personnes séropositives ont été massivement recrutées pour faciliter les opérations et faire le trait d'union entre les équipes médicales et la population. L'utilisation des «pairs», qualifiés de «patients-experts», terme placé par غ̇ve Bureau-Point au fronton de l'ouvrage, était devenue entre-temps une innoPVVIH selon l'expression consacrée) ont été recrutés intensivement dans les programmes internationaux et nationaux, renouvelant complètement le paysage des soins et de la prise en charge. Ils

Vignette (Photo (c) Inserm/Institut Curie/Raphaël Gaudin et Phillipe Bernaroch). ${ }^{1}$ Voir, par exemple, J. Léonard [1] et tout le débat influencé par Michel Foucault sur la « médicalisation » dans les sociétés occidentales. vation établie, élevée au rang de norme globale. La réaction rapide a porté ses fruits en termes de baisse de l'incidence des nouveaux cas et de mise en place d'un système de prise en charge de la maladie à VIH suffisamment efficace pour que l'on parle de Success Story dans les discours officiels.

Pendant une dizaine d'années, de 2005 à 2010, Ève Bureau-Point a mené une enquête en tant qu'anthropologue sur l'avènement des 
patients experts au Cambodge dans la lutte contre le Sida dans les hôpitaux «publics» et les hôpitaux «humanitaires», où interviennent les $0 N G$. Son analyse sur le terrain auprès des patients et des soignants l'a amenée à un regard mitigé sur processus et résultats. Elle montre en particulier comment l'embauche des patients experts a moins résulté d'un élan vital venu d'en bas, que de la mise en place, d'en haut, dans un pays exsangue après tant de catastrophes, de normes rodées à l'extérieur du pays et assimilables à une sorte de démocratisation forcée, rassurante pour la diplomatie étrangère.

En dépit du qualificatif pompeux d'experts, les patients, après une formation accélérée, ont surtout servi de petites mains. Dans un pays où l'analphabétisme est très élevé, ils assurent des fonctions multiples dans les équipes, mais n'en sentent pas moins peser sur eux une épée de Damoclès : leur emploi dépend des bailleurs de fonds et des ONG étrangères qui ont pour le moment pignon sur rue, mais ne se maintiendront pas nécessairement sur le long terme.

Ève Bureau-Point commente la façon dont les Cambodgiens accueillent la maladie et les coups du destin : ils ne réagissent pas nécessairement comme le prévoient les différents courants de la psychologie occidentale. À ce propos, elle pose la question anthropologique, douée de résonance bien au-delà du contexte cambodgien, de la nature et de la valorisation des apprentissages des patients experts. Cet apprentissage résulte à la fois de leur propre expérience de la maladie et des vicissitudes qui lui sont liées, et des enseignements reçus, lesquels ont été très variables: ils vont de l'apprentissage de la psychologie occidentale sur les suites de traumatismes à des formations centrées sur l'éducation thérapeutique ou l'étude simplifiée du savoir scientifique sur la maladie virale, sa prévention, sa transmission, et son traitement. L'anthropologue montre à quel point cet enseignement fait finalement peu de place à l'expérience propre du malade promu counsellor auprès des nouveaux venus. D'autre part, le patient élevé au rang de mentor n'est pas nécessairement reconnu comme un maître par le milieu. Tout ceci explique pourquoi la promotion des patients experts au Cambodge n'a pas créé toutes les conditions d'une médiation efficace, dans une démocratie qui reste balbutiante. La stratégie de survie domine souvent les préoccupations quotidiennes des gens, l'expérience de l'histoire enseigne aux Cambodgiens avant tout la prudence et l'acceptation des règles temporaires du jeu, bref, on assiste à une gouvernance de décor, sans instauration d'un dialogue véritable ou naissance d'un contre-pouvoir dans la société civile.
Le terme de patient-expert a été préféré par l'auteur à celui de «pair», employé le plus souvent jusque-là, qui désignait particulièrement les personnes séropositives pour le VIH, chargées d'approcher, de «sensibiliser » des acteurs méfiants et stigmatisés au sein de la société. L'accent était mis sur la familiarité avec le milieu et la capacité d'y agir comme un levain dans la pâte ou un poisson dans l'eau. Le terme de patient-expert impliquait la reconnaissance d'un savoir profane, d'une expérience unique contribuant à une meilleure connaissance des besoins des patients. Mais de quel savoir s'agit-il vraiment et comment peut-il influencer les décisions locales? La question clairement posée reste en définitive en suspens, et il n'y a donc pas lieu de s'étonner que ce savoir mal défini ne s'articule pas facilement à un pouvoir, au-delà des proclamations. Enfin, la question de l'intervention des ONG dans l'avenir (à laquelle la population semble attachée) reste également sans réponse. Elle concerne bien d'autres pays que le Cambodge.

En conclusion, un livre soulevant des questions de la plus grande actualité passionnera ceux qui ont travaillé à l'étranger ou en ont l'intention et tous ceux qui s'interrogent sur les directions actuelles de la santé globale ou santé internationale soumise à un modèle unique, et sur les réalités à découvrir derrière une terminologie et une stratégie proposées un peu vite comme valides et efficaces universellement. $\diamond$

The expert patients in the fight against AIDS

in the Cambodia

\section{LIENS D'INTÉRÊT}

L'auteur déclare n'avoir aucun lien d'intérêt concernant les données publiées dans cet article.

\section{RÉFÉRENCES}

1. Léonard J. La médecine entre les savoirs et les pouvoirs. Paris : Aubier 1992.

2. Bureau-Point $\varepsilon$. Les patients experts dans la lutte contre le Sida au Cambodge. Anthropologie d'une norme globalisée. Préface Alice Desclaux. Aix-en-Provence : Presses Universitaires de Provence, 2016 : 250 p.

\section{TIRÉS À PART}

A.M. Moulin

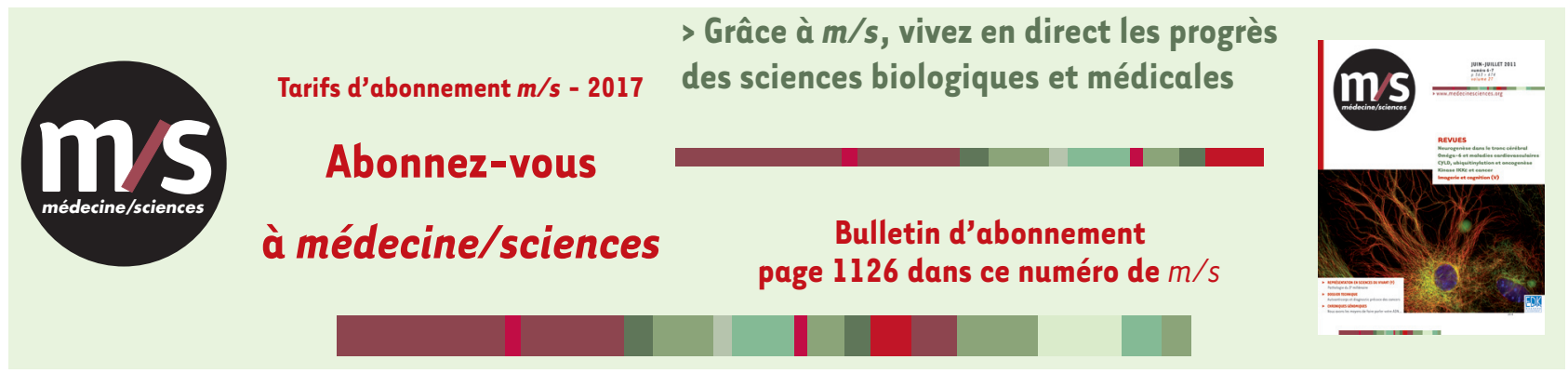

